\title{
The Structure and Function of Bacterial Actin Homologs
}

\author{
Joshua W. Shaevitz ${ }^{1}$ and Zemer Gitai $^{2}$ \\ ${ }^{1}$ Department of Physics and the Lewis-Sigler Institute for Integrative Genomics, Princeton University, \\ Princeton, New Jersey 08544 \\ ${ }^{2}$ Department of Molecular Biology, Princeton University, Princeton, New Jersey 08544 \\ Correspondence: zgitai@princeton.edu
}

During the past decade, the appreciation and understanding of how bacterial cells can be organized in both space and time have been revolutionized by the identification and characterization of multiple bacterial homologs of the eukaryotic actin cytoskeleton. Some of these bacterial actins, such as the plasmid-borne ParM protein, have highly specialized functions, whereas other bacterial actins, such as the chromosomally encoded MreB protein, have been implicated in a wide array of cellular activities. In this review we cover our current understanding of the structure, assembly, function, and regulation of bacterial actins. We focus on ParM as a well-understood reductionist model and on MreB as a central organizer of multiple aspects of bacterial cell biology. We also discuss the outstanding puzzles in the field and possible directions where this fast-developing area may progress in the future.

$\mathrm{T}_{\mathrm{t}}^{\mathrm{h}}$ he discovery of cytoskeletal proteins in bacteria has fundamentally altered our understanding of the organization and evolution of bacteria as cells. Homologs of eukaryotic actin represent the most molecularly and functionally diverse family of bacterial cytoskeletal elements. Recent phylogenetic studies have identified more than 20 subgroups of bacterial actin homologs (Derman et al. 2009) (Fig. 1). Many of these bacterial actins are encoded on extrachromosomal plasmids, but most bacterial species with nonspherical morphologies also encode chromosomal actin homologs (Daniel and Errington 2003). The two earliest proteins to be characterized as bacterial actins were the chromosomal protein MreB (Jones et al. 2001) and the plasmidic protein ParM (Jensen and Gerdes 1997). MreB and ParM remain the bestcharacterized of the bacterial actins and we will thus focus on these two proteins for most of this article.

The appreciation that bacteria possess actin homologs only occurred in the past decade. MreB was first identified as a protein involved in cell shape regulation in Escherichia coli in the late 1980s (Doi et al. 1988). In the early 1990s, pioneering bioinformatic studies identified similarities in a group of ATPases that have five conserved motifs (Bork et al. 1992), a feature dubbed the actin superfamily fold. Although

Editors: Lucy Shapiro and Richard Losick

Additional Perspectives on Cell Biology of Bacteria available at www.cshperspectives.org

Copyright (C) 2010 Cold Spring Harbor Laboratory Press; all rights reserved; doi: 10.1101/cshperspect.a000364

Cite this article as Cold Spring Harb Perspect Biol 2010;2:a000364 
J.W. Shaevitz and Z. Gitai

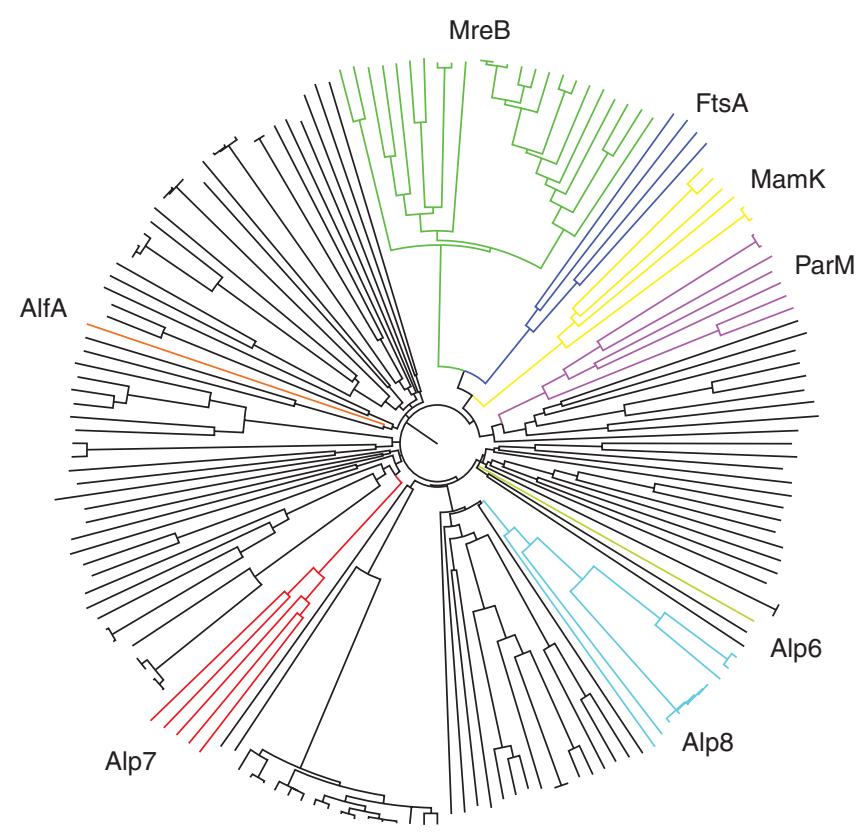

Figure 1. The superfamily of bacterial actin homologs. Shown is a phylogenetic tree of the bacterial actin subfamilies that have been identified to date based on sequence homology. The subfamilies that have been experimentally shown to polymerize are labeled and colored. (Courtesy of Joe Pogliano, based on Derman et al. 2009.)

this group includes actin and MreB, it also contains proteins that do not polymerize into filaments, such as sugar kinases like hexokinase and chaperones like Hsp70. A number of bacterial proteins are present in the actin superfamily, including the bacterial cell division protein FtsA which interacts with the tubulin homolog FtsZ and may or may not form filaments in different contexts (van den Ent and Lowe 2000). Because MreB did not appear significantly more related to actin than these nonfilamentous proteins, the weak sequence similarity with actin was largely ignored for the better part of a decade. This changed in 2001 when two seminal papers showed that Bacillus subtilis MreB forms cytoskeletal filaments in vivo (Jones et al. 2001) and that Thermotoga maritima MreB forms cytoskeletal filaments in vitro (van den Ent et al. 2001). Indeed, structural and biochemical studies of both MreB and ParM have convincingly showed that these proteins closely resemble actin and polymerize into linear filaments in a nucleotidedependent manner (Fig. 2).
Research following the identification of bacterial cytoskeletal proteins has focused on understanding their assembly, regulation, and function. Here, we will summarize our current understanding of these issues and highlight the outstanding questions. We will begin with ParM, whose well-characterized assembly and dynamics represent a model for future studies of all cytoskeletal proteins. We will then focus on $\mathrm{MreB}$, whose diverse activities appear to be central to the cell biology of many bacterial species.

\section{PARM AS A MODEL FOR STUDYING BACTERIAL ACTINS}

Perhaps the most completely understood bacterial actin is ParM, the plasmid segregating protein from the R1 plasmid. An interdisciplinary effort by a number of groups over the last decade has illuminated the details of ParM function from its fundamental molecular properties to a reconstitution of plasmid segregation in an 

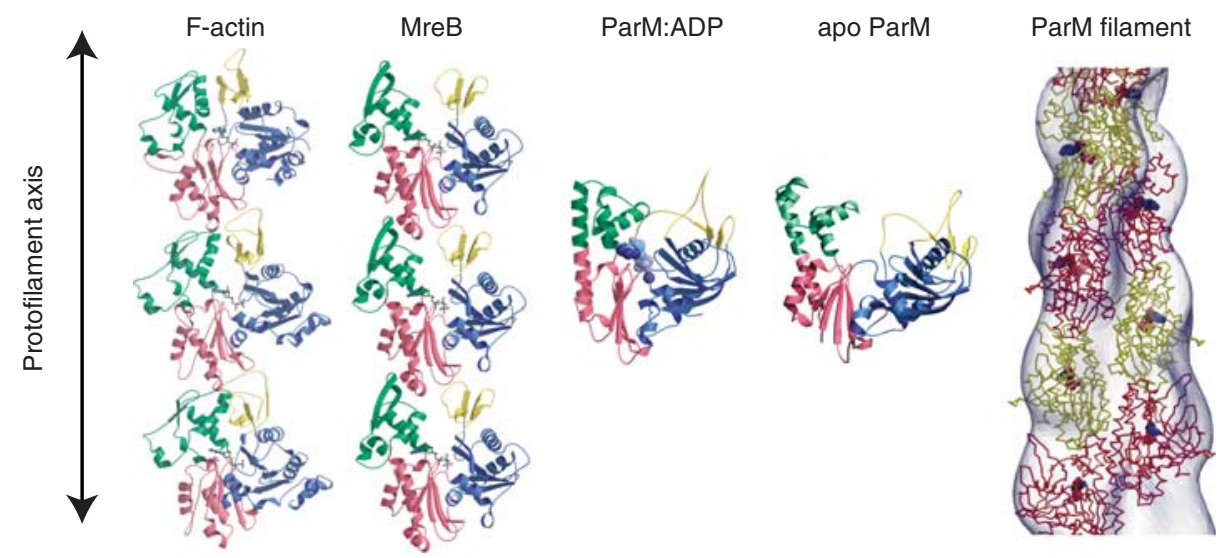

Figure 2. Structures of F-actin (Holmes et al. 1990), MreB (van den Ent et al. 2001), and ParM (van den Ent et al. 2002). (Left) Structures of F-actin filaments (PDB entry 1YAG). (Second from the left) MreB filaments from T. maritima (PDB entry 1JCE). (Center) ParM:ADP monomer in the "closed" conformation. (Second from the right) apo ParM monomer in the "open" conformation. (Right) ParM filament. Shown are the position of the nucleotide within the interdomain cleft, the conservation of fold, and the axis of the protofilament extension (arrow). Note that the conformational change shown for ParM from the "open" to "closed" state is predicted for all actin homologs. (Adapted, with permission from, Michie and Löwe 2006.)

artificial system. This multipronged approach brings together techniques from biochemistry, molecular biology, imaging, and materials science to provide an integrated solution to how ParM filaments form and how they lead to the movement of DNA. It is likely that this kind of approach will be useful in the study of the other, lesser-understood bacterial actins.

\section{ParM Function and Mechanism}

Plasmids are naturally occurring molecular parasites that often exploit their host cells' machinery for replication but provide their own segregation machinery. By 1997 it was known that the low-copy number E. coli plasmid R1 is actively partitioned during cell division so that each daughter cell retains the drug resistance conferred by the plasmid (Jensen and Gerdes 1997). This partitioning is achieved by the active positioning of two R1 sister plasmids to opposite ends of a cell during cell division (Jensen and Gerdes 1997). The machinery that directs the two plasmids to opposite cell poles is grouped in a locus termed par. The presence of this operon in a plasmid lowers the frequency of plasmid loss during division by several orders of magnitude (Gerdes et al. 1985). The Par machinery consists of three parts: a cis-acting region of DNA, parC, that acts as a centromere; the ParR protein that binds to 10 repeats within $\operatorname{par} C$ and has kinetichore-like activity; and the actin-homolog ParM, originally labeled as the partitioning motor, which drives segregation through polymerization dynamics (Dam and Gerdes 1994).

The details of plasmid segregation are elegant both in their simplicity and their efficiency. Throughout the cytoplasm, ParM filaments actively lengthen and shorten in a process known as dynamic instability that somewhat mimics the action of spindle microtubules (Garner et al. 2004). However, when the ParM filaments are bound to a plasmid via the ParR/parC complex they are stabilized against depolymerization (Garner et al. 2004). Consequently, plasmid bound ParM filaments proceed to elongate as much as they can, which in a rod-shaped cell pushes the plasmids to the cell poles at opposite ends of the cell axis (Garner et al. 2007) (Fig. 3).

This mechanism was discovered through an interdisciplinary approach to the study of plasmid segregation. Imaging work in vivo first 

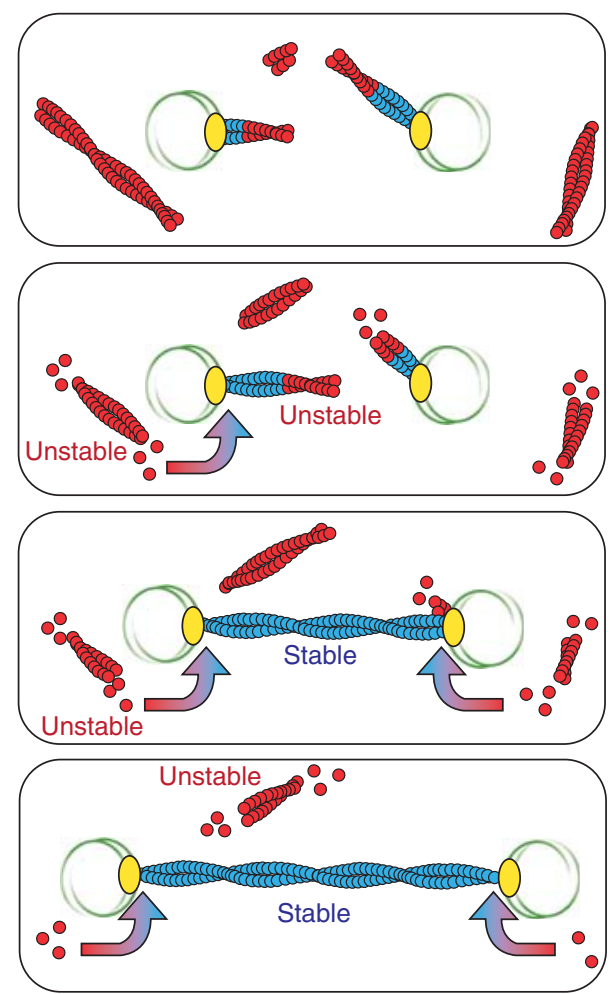

Figure 3. The assembly dynamics of ParM drive R1 plasmid segregation. Free ParM filaments ends (red) are dynamically unstable and undergo catastrophe. ParR (yellow) associates with the parC loci on the R1 plasmid (plasmids are green). Plasmid-bound ParR captures and stabilizes ParM filaments (blue). When both ends of a ParM filament are stabilized by ParR, a productive spindle is formed. Insertional polymerization at the filament ends serves to drive the two plasmids apart. (Adapted, with permission, from Garner 2008.)

defined the segregation pattern of plasmids and showed that the ParM protein forms long filaments in cells (Moller-Jensen et al. 2002; Moller-Jensen et al. 2003). Mutagenesis and other molecular techniques led to the mapping of the par locus (Dam and Gerdes 1994). In vitro work, including structural, biochemical, and imaging experiments confirmed the initial hypotheses from in vivo experiments and allowed for the reconstitution of the entire segregation process (Moller-Jensen et al. 2003; Garner et al. 2004; Garner et al. 2007). Richard Feynman once said that "What I cannot create, I do not understand." It is in this spirit that we think that ParM is well understood. In the paragraphs to follow, we discuss in greater detail what is currently known about ParM from the molecular to the cellular scales.

\section{ParM Polymerization and Structure}

ParM forms filaments both in vivo and in vitro. Initial immunofluorescence imaging showed that ParM formed long, curved filaments (Moller-Jensen et al. 2002). These authors observed that ParM filaments often spanned the length of rod-shaped cells and that the filaments were gently curved. Interestingly, the length of the filaments was observed to be dynamic. In vitro, purified ParM was shown to polymerize into long, straight filaments. Polymerization requires ATP, or the nonhydrolyzable ATP analogs ATP $\gamma S$ and AMPPNP (Moller-Jensen et al. 2002). In the presence of ADP, ParM does not polymerize. This observation, together with the dynamic nature of ParM filaments in cells, led to a dynamic instability model for plasmid segregation, similar to that for tubulin, where ATP-bound ParM monomers bind stably to the end of ParM filaments whereas posthydrolysis, ADP-bound monomers lead to depolymerization from the filament end.

The three-dimensional crystal structure of monomeric ParM shows remarkable similarity to that of actin (Fig. 2). ParM is an asymmetric protein with a barbed and a pointed end made up of four domains: IA, IB, IIA, and IIB, which respectively correspond to the actin domains 1 , 2, 3, and 4 (van den Ent et al. 2002). ParM has been successfully crystallized in the nucleotidefree and ADP bound states. These structures show that the nucleotide binds in the interdomain cleft of the barbed end. On nucleotide binding, a rigid-body rotation of about $25^{\circ}$ of domains I and II closes the cleft slightly. This conformational change is thought to cause the nucleotide-induced depolymerization of ParM filaments.

The structure of ParM filaments was solved by docking the monomeric crystal structures into three-dimensional electron microscopy images of filaments (van den Ent et al. 2002). 
Filaments assemble with the pointed end of one monomer enclosed by the barbed-end of the next. Along the filament, adjacent monomers are rotated by about $166^{\circ}$ and translated by 2.5 $\mathrm{nm}$ from each other. Even though this is a slightly tighter twist and shorter axial spacing than that of actin, ParM still forms a twisted, two-start helix. One significant difference between actin and ParM filaments is that the ParM helix is left-handed whereas actin is righthanded. The juxtaposition of a similar but chirally opposite geometry suggests that this helical shape might afford the filaments a high level of stability that has been arrived at independently through convergent evolution. Similar conclusions about the shape of ParM filaments in vivo have been made using high-resolution cryo-EM tomography (Salje et al. 2009). More recently, analysis of additional bacterial actin homologs supports the idea that though these proteins all polymerize, the details of their filament formation can be quite divergent (Polka et al. 2009). Although the sequence and 3D structure of the ATPase domains of ParM have significant homology with actin, the exposed residues of ParM are very different in their chemical properties from those of actin. Consequently, proteins that bind to actin and to ParM are not expected to share much similarity.

\section{ParM Biochemistry and Biophysics}

In vitro experiments have been very successful at verifying and enhancing the early models of plasmid segregation derived from in vivo experiments. Jensen and Gerdes showed that mutation of the aspartate at position 170 of ParM abolished both the protein's ATPase activity and its ability to segregate plasmids in vivo (Jensen and Gerdes 1997; Jensen and Gerdes 1999). Immunofluorescence imaging showed that these mutants showed hyperfilamentation, lending support to the idea that ParM dynamics are required for proper plasmid segregation.

Right-angle light scattering measurements have been used to measure the kinetics of ParM filament polymerization. Moller-Jensen and others showed that after an initial bout of polymerization, ParM slowly hydrolyzes bound
ATP leading to filament depolymerization (Moller-Jensen et al. 2002). Additional ATP added after this depolymerization phase causes a new round of fast polymerization and slow depolymerization, indicating that the monomers are capable of going through multiple rounds of nucleotide stimulated polymerization and depolymerization. Most interestingly, filaments are stabilized by the addition of both ParR and parC. When these three components are mixed together, filaments do not depolymerize and the critical concentration for polymerization is reduced (Garner et al. 2007). These results were confirmed by subsequent FRET measurements that verified the nucleation condensation mechanism of ParM polymerization. Garner and coworkers found a critical nucleus size of three monomers that defines the two-stranded helical filament geometry (Garner et al. 2004).

Although light scattering is a useful tool to measure the typical size of an ensemble of oligomers in solution, it cannot resolve the nature of individual filament dynamics. For example, it can be difficult to distinguish whether some monomers are incapable of forming polymers or if the ensemble of polymers is very dynamic. Garner et al. used total-internal fluorescence microscopy to directly visualize single Alexadye labeled ParM filaments in vitro (Garner et al. 2004). Using a two-color assay in which red-labeled monomers were added to greenlabeled filament seeds, they were able to distinguish growth from the two filament ends. Using the nonhydrolyzable substrate AMPPNP, they observed steady growth from both ends of long, stable filaments. In the presence of ATP, however, filaments grew symmetrically for a while but then rapidly depolymerized to completion from one end. The time scales associated with the filament growth and shrinking were consistent with a mechanism in which an ATP cap stabilizes the ParM filament. If the terminal monomers hydrolyze their bound nucleotide, the filament becomes unstable. These studies thus showed that in contrast to actin, which preferentially polymerizes at one filament end at a constant rate, ParM polymerizes symmetrically and experiences dynamic instability with 
bouts of steady polymerization interrupted by rapid depolymerization.

Putting all of these ideas together, Garner and colleagues showed that ParM, ParR, and parC are sufficient to reconstitute plasmid segregation in vitro (Garner et al. 2007). The authors coated small, 350-nm diameter beads with a piece of DNA that contained the parC sequence. When added to a solution of ParR and fluorescently labeled ParM, the beads formed aster-like clouds of highly dynamic ParM. When two beads were in proximity, their ParM structures stabilized one another to generate long spindle-like structures. EM images indicated that single filaments extended all the way from one bead to the other, suggesting that the long structures resulted from stabilization of both ends of the ParM filaments. These filaments were able to elongate by insertional polymerization between the tips of the filaments and the beads, thereby pushing the DNAcoated beads farther and farther apart (Fig. 3). The energetics of this elongation were proposed to be driven by a monomer excess generated by the dynamic instability of the nonstabilized ParM. Most excitingly, when the parC-coated beads, ParM, and ParR were placed in long microfabricated channels, the ParM spindle elongated along the long axis of the channel, thereby breaking symmetry to separate pairs of beads to opposite ends of the cylinder.

Although the ability to reconstitute the entire plasmid segregation process is nothing short of remarkable, it is worth noting that it shows sufficiency for how the system could work, but does not prove how it does work in vivo. Thus, several exciting questions about ParM remain to be answered. For example, does the rest of the plasmid DNA really just play a passive role in the whole process? Also, why is it that ParM and actin filaments are structurally similar yet one polymerizes symmetrically at both ends while the other polymerizes asymmetrically? Along similar lines, EM studies both in vitro and in vivo suggest that both ends of the filament associate with the ParR/parC complex (Garner et al. 2007; Salje et al. 2009), and work by Choi and colleagues used very small gold beads to show that in a ParM spindle, a single copy of parC is bound to the end of a single ParM filament (Choi et al. 2008). So how does the $\mathrm{ParR} /$ parC manage to interact with both ends of an asymmetric polymer? Other unresolved issues surround the nature of the dynamic instability and the ability of other nucleotides such as GTP to stabilize ParM filaments (Popp et al. 2008). Nevertheless, ParMmediated plasmid segregation remains a prototype for how combining genetics, cell biology, biochemistry, and biophysics can lead to an emergent understanding of dynamic systems.

\section{THE LOCALIZATION AND FUNCTION OF MREB}

ParM is encoded by a subset of plasmids and carries out one highly specific function, plasmid segregation. In contrast, the most widely conserved bacterial actin homolog, MreB, is encoded in the chromosomes of many different species and can participate in many different cellular activities (Daniel and Errington 2003). MreB homologs have been implicated in nearly every spatially organized cellular process, including cell growth, morphogenesis, polarity, protein localization, organelle positioning, division, and differentiation, as well as chromosome segregation, replication, and decatenation (reviewed in Carballido-Lopez 2006). The current challenge is to understand the mechanism by which MreB impacts these cellular processes and distinguish its primary roles from their secondary consequences. MreB also assembles into an interesting, often helical, localization pattern that may help it execute its many functions (Jones et al. 2001). Finally, MreB clearly cannot be doing all of these things on its own; indeed, a growing number of MreB interactors are being identified. In this section we will focus on the best characterized of these MreB localizations, functions, and interactors.

\section{MreB Proteins are Relatively Diverse}

Discussion of MreB is inherently complicated because of the vast diversity of MreB homologs in the bacterial and archael kingdoms. At least one MreB homolog is found in most 
nonspherical bacteria (Daniel and Errington 2003). The exceptions to this rule include a number of plant and animal pathogens that are rod-shaped but lack a clear MreB homolog, including Mycoplasmas, Mycobacteria, and Rhizobiae. There are also several spherical bacteria such as Cyanobacteria and Planctomycetes that have MreB homologs. Many bacteria also encode multiple MreB homologs. The bestcharacterized example is $B$. subtilis, which has three MreB homologs: MreB, Mbl, and MreBH (Jones et al. 2001). The three B. subtilis MreB homologs each has similar homology to other MreBs ( $\sim 50 \%$ identity to T. maritima MreB), such that even though only one of them bears the name "MreB", they should each be viewed equivalently. Spiroplasmas have even more MreB homologs, as many of these species have five MreB proteins with more divergent sequences (Kurner et al. 2005). Yet other species, like Magnetospirillum magnetotactum, have both a relatively well-conserved $\mathrm{MreB}$ homolog and a second more divergent actin superfamily member, MamK (Komeili et al. 2006). All MreB homologs share the same basic actin superfamily signature, and all MreB homologs characterized to date have been found to be able to polymerize. Nevertheless, the diversity in number and sequence of MreB homologs means that MreB cannot be treated as a single entity and that it is important to specify which MreB homolog one is discussing in a specific context. Most of the structural and biochemical work on MreB has been performed on the T. maritima homolog, whereas the in vivo properties of MreB have best been characterized in B. subtilis, Caulobacter crescentus, and E. coli. Our discussion here will primarily focus on these systems.

\section{MreB Localization and Dynamics}

MreB localization was first characterized in $B$. subtilis for both the $m r e B$ and $m b l$ genes (Jones et al. 2001). These proteins were found to form right-handed helical structures by deconvolution microscopy. In some cells a double helix is observed, whereas others resemble a single helix. Assessing the exact dimensions and topologies of these helices remains an active area of investigation that is complicated by two issues. First, the small size of these structures is near the diffraction-limited resolution of light microscopy. Second, immunofluorescence (IF) microscopy requires fixation that can alter cell ultrastructure, whereas both aminoand carboxy-terminal fluorescent protein fusions to MreB can perturb both the function and localization of native MreB. Very recently, an internal fusion that placed mCherry in the middle of the E. coli MreB protein was found to be largely functional (Bendezu et al. 2009), suggesting that these issues, perhaps in combination with recent advances in subdiffractionlimited microscopy, might soon be resolved.

Nevertheless, in many cases the IF and the GFP-MreB images agree, leading to a consensus view that MreB prefers, at least locally, to form a helix with several turns per cell length. In B. subtilis, the three MreB homologs colocalize with an approximate pitch of $0.75 \mu \mathrm{m}$, suggesting that the three MreB isoforms may functionally interact or copolymerize into a single structure (Carballido-Lopez et al. 2006). As cells elongate, the pitch appears to remain constant with addition of new helical turns. In Caulobacter, both IF and GFP-MreB reporter studies indicate that the localization of MreB is regulated during the cell cycle (Figge et al. 2004; Gitai et al. 2004). Early in the cell cycle, Caulobacter MreB forms a patchy, potentially helical, pattern that extends from pole to pole. As the cell cycle progresses, this helix condenses into a ring at the presumptive division plane. Before cell division occurs, MreB expands from a ring back into a helical form such that each daughter cell inherits a similar polymer structure. The transition from helix to ring depends on the FtsZ tubulin homolog, the central organizer of the division machinery (Figge et al. 2004). Localization experiments in other species such as E. coli, P. aeruginosa, and Rhodobacter have also characterized both helical and medial MreB distributions (Slovak et al. 2005; Vats et al. 2009; Cowles and Gitai 2010), though the physiological consequences of this transition remain unclear.

In contrast to B. subtilis cells that colocalize three closely related MreB homologs, MreB 
does not appear to colocalize with more highly diverged actin homologs. For example, in M. magnetotactum, the divergent actin MamK forms a straight filament structure unlike any MreB localization pattern that has been characterized (Komeili et al. 2006). Similarly, plasmidic actin homologs like ParM, AlfA, and the Alp proteins form straight structures that do not colocalize with MreB (Moller-Jensen et al. 2002; Becker et al. 2006; Derman et al. 2009). Some of these proteins, including ParM and AlfA, have highly divergent polymer interaction surfaces and geometries (van den Ent et al. 2001; van den Ent et al. 2002; Polka et al. 2009), which may function to actively prevent their copolymerization with MreB.

While the entire MreB structure is dynamically rearranged in Caulobacter, it also appears that the subunits of the MreB helix are highly dynamic in all cells examined. These dynamics have been assessed by FRAP for $\mathrm{Mbl}$ (Carballido-Lopez and Errington 2003), by speckle tracking for all three MreB proteins in B. subtilis (Defeu Soufo and Graumann 2004), and by single-molecule imaging of MreB in Caulobacter (Kim et al. 2006). The Caulobacter MreB appears to have faster turnover kinetics than observed in $B$. subtilis, but it remains unclear whether this reflects species-specific differences or differences in the types of assays used (FRAP vs. single-molecule imaging). Although the Caulobacter studies suggest that individual MreB filaments may be polarly assembled (Kim et al. 2006), there does not appear to be an overall pole-to-pole polarity in the MreB helix, suggesting that the MreB helix includes individual filaments with mixed polarities.

Further evidence for the dynamic nature of MreB assembly comes from experiments with the small molecule A22 (S-(3,4-dichlorobenzyl) isothiourea). A22 was first found in a chemical genetic screen for compounds that increased the rate of chromosome loss in E. coli (Iwai et al. 2002). A22 was found to cause cells to become round, but its cellular target was unknown until A22-resistant mutations were mapped to the $m r e B$ gene, first in Caulobacter (Gitai et al. 2005), and subsequently in other species such as E. coli and P. aeruginosa (Kruse et al. 2006; Robertson et al. 2007; Cowles and Gitai 2010). In Caulobacter, A22 rapidly delocalizes MreB filaments in vivo, and the resistant mutants all map to the nucleotide-binding pocket (Gitai et al. 2005). Indeed, biochemical studies (detailed below) show that A22 functions by binding the MreB nucleotide-binding pocket and mimicking the ADP-bound low polymerization affinity monomer state (Bean et al. 2009). When A22 is applied to either purified MreB in vitro (Bean et al. 2009) or MreB that has been heterologously expressed in the eukaryote S. pombe (Srinivasan et al. 2007), A22 inhibits new MreB polymerization but does not stimulate depolymerization. Consequently, the rapid delocalization of $\mathrm{MreB}$ observed in bacterial cells likely results from dynamic MreB depolymerization that can no longer be balanced by A22-inhibited polymerization. The increased dynamics of MreB assembly in vivo also suggests that additional cellular factors exist to stimulate these dynamics. The identification and characterization of such factors will be an important area of future investigation.

Another important yet unresolved issue is the mechanism by which MreB adopts its localization pattern. MreB filaments are not obviously helical either in vitro or on heterologous expression in eukaryotes (van den Ent et al. 2001; Srinivasan et al. 2007). It is possible that MreB helices are the mechanical consequence of forcing a linear polymer into a cylindrical container. Alternatively, accessory factors may change the preferred conformation of MreB filaments in bacteria. The dynamic redistribution of MreB during the Caulobacter cell cycle supports the idea that MreB conformation and localization can be regulated. One candidate for such regulation is RodZ, which was recently characterized as a protein that binds MreB and potentially links it to the inner membrane (Shiomi et al. 2008; Alyahya et al. 2009; Bendezu et al. 2009; van den Ent et al. 2010). RodZ and MreB have an interdependent genetic relationship and similar loss-of-function phenotypes, making it difficult to dissect a linear localization hierarchy between these proteins. Yet other MreB-interacting proteins such as Ef-Tu (Defeu 
Soufo et al. 2010), Pbp2 (Figge et al. 2004), or other as-yet-unidentified proteins could also influence MreB localization.

\section{MreB is a Key Regulator of Cell Shape Determination}

MreB was first characterized as an E. coli mutant that caused normally rod-shaped cells to become spherical (Doi et al. 1988). Indeed, loss of proper cell shape has emerged as the most common defect associated with MreB proteins across most species. The central importance of MreB for achieving rodlike elongation is supported by the phylogenetic observation that MreB proteins are primarily found in nonspherical cells (Daniel and Errington 2003). However, there are exceptions to this rule. For example, Helicobacter pylori MreB has been reported to influence chromosome dynamics and virulence factor secretion without affecting cell shape (Waidner et al. 2009), and in Streptomyces coelicolor MreB affects sporulation but does not affect the shape of vegetatively growing cells (Mazza et al. 2006). To more generally survey how rodlike bacteria grow, the Errington lab chemically labeled nascent cell wall synthesis and characterized two distinct cylindrical growth modes in different species (Daniel and Errington 2003). One mode is more common and uses MreB to direct the insertion of new cell wall material along the length of the cylinder. These species generally have inert cell poles. The second mode is MreB-independent and involves insertion of new cell wall material at the cell poles. A third, FtsZ-dependent elongation mechanism has also been more recently proposed (Aaron et al. 2007).

MreB regulates cell shape in three wellcharacterized model systems: B. subtilis, E. coli, and Caulobacter. In B. subtilis, mutants in the three MreB homologs have different morphologies (Jones et al. 2001). It is possible that these MreB proteins have specialized for distinct functions. Alternatively, a study demonstrating partial redundancy between these mutants suggests that they may all influence the same process to different extents, perhaps because of differential expression levels (Kawai et al.
2009). In E. coli grown under normal conditions, cells lacking MreB become spheres and eventually lyse (Bendezu and de Boer 2008). However, the lethality of loss of MreB can be suppressed either by overexpressing cell division proteins or reducing the rate of cell growth (Bendezu and de Boer 2008). In these conditions $m r e B$ mutants are viable but accumulate intracellular vesicles, suggesting that membrane production is disregulated (Bendezu and de Boer 2008). The lethality of a mutation in one of the B. subtilis mreB genes can also be suppressed by growth in increased levels of $\mathrm{Mg}^{++}$ (Formstone and Errington 2005).

Studies from both B. subtilis and Caulobacter suggest that at least one way in which MreB influences the cell wall is by directing the insertion of new cell wall material in a helical pattern. The bacterial cell wall is composed of stiff peptidoglycan strands that are polymerized by transglycosylases and crosslinked by transpeptidases to generate a meshlike superstructure (Holtje and Heidrich 2001). The cell wall has been thought to be the pressure-bearing cell shape determinant because cell wall lysis causes cells to round up and isolated cell walls retain their general morphology (Young 2006). Because both MreB and new cell wall insertion follow helical patterns, the basic model for how MreB directs cell shape determination is that MreB helically localizes proteins that in turn lead to helical peptidoglycan assembly (Daniel and Errington 2003). It is also possible that as in eukaryotes, the MreB cytoskeleton plays a mechanical role in directing proper morphogenesis.

Consistent with the cell wall patterning model, Caulobacter MreB biochemically associates with the cytoplasmic tail of a cell wall assembly enzyme, the $\mathrm{Pbp} 2$ peptidoglycan transpeptidase, and directs its localization (Figge et al. 2004; Dye et al. 2005). Caulobacter MreB has also been proposed to direct the localization of cytoplasmic proteins that direct peptidoglycan subunit synthesis (White et al. 2010) and inhibition of MreB with the small molecule A22 leads to shortened cell wall glycan strands (Takacs et al. 2010), indicating that MreB may have a general influence on peptidoglycan assembly. In B. subtilis, the Pbp proteins adopt 
a helical localization pattern (Scheffers et al. 2004). However, single mreB mutants do not disrupt this localization (Scheffers et al. 2004), possibly because of partial redundancy between the different MreB. Unlike E. coli and Caulobacter, which are gram-negative bacteria, $B$. subtilis are gram-positive and their cell wall is also composed of teichoic acids. The proteins that assemble teichoic acids are also helically localized, though it remains unclear if this helical distribution is related to that of the MreB proteins (Formstone et al. 2008). In addition, MreBH interacts with the cell wall hydrolase LytE (Carballido-Lopez et al. 2006), and theoretical studies suggest that cell shape could be dictated by patterning either peptidoglycan insertion or cleavage (Huang et al. 2008).

In many species, $m r e B$ is found in the same operon as two other genes, mreC and mreD. $\mathrm{MreB}, \mathrm{MreC}$, and $\mathrm{MreD}$, along with the cell wall assembly proteins $\mathrm{Pbp} 2$ and RodA have been proposed to form a complex that collaborates to coordinate cell elongation (Kruse et al. 2005). This complex of predominantly transmembrane proteins may explain how the cytoplasmic MreB structure can have such an impact on the assembly of the cell wall, which occurs outside of the inner membrane. In Caulobacter, MreC is cleaved and released into the periplasm where it forms a helical structure whose assembly is independent of MreB (Divakaruni et al. 2005). The crystal structure of $\mathrm{MreC}$ suggests that it may form polymers and could thus act as a periplasmic cytoskeleton (van den Ent et al. 2006). Further genetic and colocalization studies in Caulobacter suggest that MreC acts to localize peptidoglycan assembly in the periplasm (Divakaruni et al. 2005; Dye et al. 2005), whereas MreB acts to localize peptidoglycan precursor synthesis in the cytoplasm (Figge et al. 2004; White et al. 2010). However, this model must either be oversimplified or species-specific because some species that have MreB lack MreC or vice-versa, and in yet other species, MreB localization depends on MreC (Kruse et al. 2005).

The growing body of evidence that MreB can influence nearly every aspect of cell wall assembly suggests either that all of these processes are highly interdependent or that MreB has multiple independent roles in regulating cell shape. Dissecting the specific roles of MreB in this process has been complicated by the fact that we do not have very good tools for studying peptidoglycan structure and dynamics. Fluorescent derivatives of antibiotics that target nascent peptidoglycan structures have proved useful (Daniel and Errington 2003; Tiyanont et al. 2006), but can also perturb cell wall assembly and occasionally produce conflicting results. Recent studies have used high-resolution atomic force microscopy to study the exposed cell walls of gram positives or isolated gram-negative cell walls (Yao et al. 1999; Touhami et al. 2004), and an elegant electron cryotomography study gave the first glimpses into the structure of Gramnegative cell walls (Gan et al. 2008). By combining these new approaches for studying peptidoglycan, the next few years promise to reveal a great deal about the assembly and regulation of bacterial cell walls.

\section{MreB Regulates Protein Localization}

MreB has also emerged as a key regulator of the subcellular organization of bacterial proteins. The role of MreB in polar protein localization was first characterized in Caulobacter, where MreB was found to be necessary for the localization of multiple polar protein markers (Gitai et al. 2004). Subsequently, MreB has been implicated in a wide range of protein localization processes in many experimental systems. In addition to the proteins involved in cell wall assembly discussed in the previous section on cell shape, these include chemotaxis receptors in E. coli (Shih et al. 2005), gliding motility proteins in Myxococcus xanthus (Mauriello et al. 2010), and pilus-associated proteins in Pseudomonas aeruginosa (Cowles and Gitai 2010). There is also evidence that bacterial actins are important for localizing larger protein complexes or organelles. For example, MamK is essential for magnetosome localization in $M$. magnetotactum (Komeili et al. 2006). Similarly, MreB influences stalk assembly and localization in Caulobacter (Wagner et al. 2005; Divakaruni et al. 2007), pilus assembly in P. aeruginosa and 
M. Xanthus (Cowles and Gitai 2010; Mauriello et al. 2010), inclusion body localization in E. coli (Rokney et al. 2009), and carboxysome localization in Synechoccus elongates (Savage et al. 2010). These larger structures may particularly benefit from cytoskeletal-mediated localization because of their inherently reduced rates of diffusion.

One issue with studying MreB's functions is that MreB is pleiotropic, such that it can be difficult to untangle the direct effects of MreB on subcellular localization from indirect effects caused by decreased growth or disrupted morphology. One advance that has been of immense help in this area has been the discovery of small molecules, such as A22, that rapidly perturb MreB. The rapid action of these compounds helps temporally uncouple primary and secondary consequences of MreB disruption. For example, although MreB can be delocalized in as little as $30 \mathrm{~s}$ with these agents, the cell shape defects induced on $\mathrm{MreB}$ disruption require hours of new growth to manifest, producing a time window during which MreB is disrupted but cell shape is still unaffected (Gitai et al. 2005). One concern with using any small molecule inhibitor is the degree of specificity for the target protein of interest. Although A22 appears to primarily affect MreB, genetic studies from both $E$. coli and $P$. aeruginosa suggest that it may have additional off-target effects (Cowles and Gitai 2010; Takacs et al. 2010). Recently, two additional MreB antagonist compounds, CBR-4830 and MP265 have been characterized (Robertson et al. 2007; Takacs et al. 2010). By combining comparisons of multiple distinct MreB inhibitors and the proper use of drugresistant control strains, small molecule antagonists remain the premier method for dissecting MreB function.

Although MreB is clearly important for protein localization, and this role may explain how MreB can affect so many cellular processes, the mechanism by which MreB directs protein localization remains mysterious. Increasing evidence suggests that MreB may often be involved in the initiation, but not the maintanence, of protein localization. For example, in Caulobacter, the polar marker PopZ localizes to one cell pole early in the cell cycle and later redistributes to the other cell pole (Bowman et al. 2008). Although MreB is not required for maintaining the localization of PopZ at the initial pole, it is required for relocalizing PopZ to the second pole (Bowman et al. 2008). By treating cells with A22 before or after the induction of a fluorescent fusion to a protein of interest, one can directly distinguish whether MreB is involved in maintaining the localization of old protein (which would be delocalized regardless of whether A22 is administered before or after induction), or whether MreB is involved in initiating new protein localization (which would only be delocalized when A22 is administered before induction). Consistent with the PopZ example, MreB is also required for the initiation of $\mathrm{Pbp} 2$ localization in Caulobacter (Dye et al. 2005). These functions can also be regulated, as seen in P. aeruginosa, where MreB is required for both the initiation and maintenance of polar PilT localization when cells are grown in liquid media but is only required for initiation of PilT localization when cells are grown on solid surfaces (Cowles and Gitai 2010). Thus, although several studies have taken a protein's persistent localization in the presence of A22 as indication of MreB-independent localization, such experiments only address the importance of MreB for the maintenance of protein localization. A better understanding of both the proteins that directly interact with MreB and the singlemolecule motions of these target proteins should lead to a better understanding of the molecular mechanism by which MreB directs the dynamics of initiating protein localization.

\section{MreB and Chromosome Dynamics}

Bacterial actins have been implicated in regulating the organization of bacterial DNA. This function is clearest for the plasmidic actins such as ParM and AlfA, as discussed earlier. MreB proteins have also been implicated in chromosome dynamics, though their specific functions and mechanisms remain unclear. In E. coli, a dominant-negative MreB mutant was shown to perturb chromsome segregation (Kruse et al. 2003), and A22 treatment was found to both 
affect chromosome segregation and increase the rate of chromosome loss (Iwai et al. 2002; Kruse et al. 2006). A mechanism for this effect was suggested by the interaction of MreB with RNA polymerase, which could serve as a motor to push the two chromosomes apart (Kruse et al. 2006). In contrast, recent studies have shown that E. coli can survive and presumably segregate its chromosomes in the absence of MreB when cell growth is slowed (Bendezu and de Boer 2008), and can segregate its chromosomes when MreB localization is aberrant (Karczmarek et al. 2007). Similarly, MreB has been implicated in chromosome segregation in B. subtilis in some cases but not others (Soufo and Graumann 2003; Formstone and Errington 2005), though a careful analysis in the absence of all three MreB homologs has yet to be completed. Experiments in Vibrio cholerae and Helicobacter pylori support the role of MreB in chromosome segregation (Srivastava et al. 2007; Waidner et al. 2009), whereas the nonessential nature of MreB in other species such as S. coelicolor suggest that MreB is not necessary for segregation in these organisms (Mazza et al. 2006; Hu et al. 2007). Some insight into this apparent paradox might be gleaned from studies in Caulobacter. Under certain environmental conditions such as growth on agarose pads, the segregation of the region of the chromosome near the origin of replication is either blocked or significantly delayed by treatment with A22 (Gitai et al. 2005). However, under other conditions, such as growth in liquid media, A22 treatment only results in a mild delay in the onset of segregation, which can be largely attributed to a delay in the onset of DNA replication (Shebelut et al. 2009). Genetic studies suggest that there are multiple pathways that contribute to the process of segregation (Shebelut et al. 2009). It is possible that these pathways may act redundantly in some conditions, but that each may become essential under other, perhaps stressful, conditions. Although it remains unclear whether MreB directly or indirectly affects chromosome segregation, viewing segregation as a sequence of distinct processes may help define the specific mechanisms that collaborate to govern segregation in different contexts.
MreB has also been implicated in aspects of chromosome dynamics other than segregation. In $V$. cholerae, MreB perturbations affect chromosome condensation (Srivastava et al. 2007). Meanwhile, in E. coli, MreB was found to regulate chromosome decatenation (Madabhushi and Marians 2009). This effect appears to be direct, as in vitro studies showed that the activity of Topoisomerase IV was stimulated by purified MreB polymers and inhibited by purified MreB monomers. Finally, MreB may play a role in DNA replication, as A22 treatment slows replication in Caulobacter (Shebelut et al. 2009) and MreB can affect the positioning of DNA replication proteins in $B$. subtilis (Defeu Soufo and Graumann 2005). MreB may also be required for the replication of foreign DNA in bacteria. MreB is required for the replication of a number of phages that infect different bacterial species, and MreB specifically mediates the localization of phage replication proteins for the B. subtilis phage 29 (Munoz-Espin et al. 2009).

\section{MreB May be Important for Bacterial Pathogenesis}

The study of bacterial cell biology in general and bacterial actins in particular is rapidly advancing our understanding of the fundamentals of cellular organization and dynamics. Meanwhile, these studies also promise to yield exciting advances in our ability to combat infectious diseases. MreB is essential for the rapid growth of many bacteria, such that small molecule inhibitors of MreB could represent powerful broad-spectrum antibiotics. Recent studies have begun to suggest that MreB may also have been co-opted by pathogenic bacteria to organize their virulence mechanisms. In P. aeruginosa, MreB regulates the polar assembly of type IV pili, which are important for virulence (Cowles and Gitai 2010). In H. pylori, MreB regulates the secretion of virulence factors (Waidner et al. 2009), and Vibrio parahaemalyticus differentially regulates MreB expression on interaction with its host (Chiu et al. 2008). Bdellovibrio bacteriovorus is a bacterium that infects other bacteria, and perturbing B. bacteriovorus 
MreB perturbs this ability (Fenton et al. 2010). Consequently, understanding bacterial actins may help understand and ultimately develop both broad and narrow spectrum approaches to combating bacterial pathogenesis.

\section{MREB STRUCTURE AND ASSEMBLY}

Like ParM, MreB shares significant sequence homology with actin. An early search of known bacterial sequences indicated that MreB was a likely member of the actin superfamily based on its catalytic core (Bork et al. 1992). By sequence, MreB is the most closely related to actin of all the actin family proteins although small differences between MreB and actin exist in both the nucleotide binding site and the residues that form the monomer-monomer interface within a protofilament. The differences at the monomer-monomer interface are interesting because they must have evolved concomitantly to retain the polymerization. In vitro polymerization of MreB and subsequent solution of the polymer crystal structure was performed in 2001 by van den Ent, Amos, and Lowe (van den Ent et al. 2001). Using purified MreB1 from T. maritima, they found that polymers formed in a variety of conditions and required ATP or GTP.

MreB was the first actin for which the polymeric crystal structure was solved (van den Ent et al. 2001), as opposed to conventional actin for which only EM-based polymer structures exist (Oda et al. 2009). This breakthrough revealed a two-domain, $\mathrm{V}$-shaped configuration with the nucleotide bound in the interdomain cleft, in good agreement with models of actin filaments. The lateral spacing of monomers along an MreB protofilament is $5.1 \mathrm{~nm}$ and a high concentration of hydrophobic residues lies at the monomer-monomer interface, producing a strong binding interaction. Unlike actin and ParM, however, MreB appears to form straight protofilaments and does not assemble into two-filament twisted helices. The ability to express, purify, polymerize, and crystallize recombinant MreB polymers provides the potential to study structural properties of filamentous proteins in a way that has not been possible with actin or tubulin and should result in some major breakthroughs in the near future.

\section{MreB Filament Structure and Biophysics}

Although MreB appears to form a helical structure in cells, in vitro MreB usually forms very straight and stiff filaments, and sometimes tight, ringlike spirals depending on the polymerization conditions (van den Ent et al. 2001; Esue et al. 2005; Esue et al. 2006). The MreB protofilament is straight with a longitudinal spacing of $5.1 \mathrm{~nm}$. These protofilaments associate laterally to build thick, crystalline bundles. Bean and Amann used fluorescent imaging to examine Alexa-fluor-labeled Cys332MreB and observed thick bundles $\sim 3$ microns in length that appeared very rigid because of their lack of thermally driven bending fluctuations (Bean and Amann 2008). Similar features were observed when a GFP-labeled MreB was expressed in fission yeast (Srinivasan et al. 2007). Esue et al. (2006) measured the bulk rheological properties of MreB gels (Esue et al. 2006). At physiological concentrations, the MreB formed a very stiff gel $\left(\sim 10 \mathrm{dyn} / \mathrm{cm}^{2}\right)$ in about $2 \mathrm{~min}$. However, these types of bulk measurements are difficult to interpret and the relationship between gel stiffness and MreB filament function remains to be explored. Very recent measurements have shown that MreB contributes significantly to the overall stiffness of E. coli cells (Wang et al. 2010).

\section{MreB Biochemistry}

A consensus view of the in vitro kinetics of MreB polymerization has been more difficult to measure than for ParM because different experimental groups have produced varying results using different MreB constructs. To date, the biochemical mechanisms of MreB polymerization and dynamics remain an active area of research.

Esue and coworkers published the first measurements of MreB polymerization kinetics in 2005 (Esue et al. 2005). Using a His-tagged form of T. Maritima MreB1, the authors found that polymerization was strongly dependant on 
temperature and the concentrations of different ions in solution. They measured a critical concentration of $3 \mathrm{nM}$, a hundred times smaller than that for actin. Further experiments showed that MreB can use ATP or GTP as a substrate equally well, unlike other proteins such as actin (Esue et al. 2006).

Work in 2008 from Bean and Amann focused on the same protein but measured a different behavior (Bean and Amann 2008). These authors purified the native form of T. Maritima MreB1 without a His-tag through ion exchange and gel filtration chromatography and differential centrifugation. They found two phases of polymerization, one that uses divalent cations and one that does not. These phases have been hypothesized to correspond with the nucleation and elongation phases of polymer growth. Their more "native" protein was also less temperature sensitive than those reported previously. ATP hydrolysis of the enzyme was fast, implying that in vivo most polymerized $\mathrm{MreB}$ is in an ADP-bound state. In addition, the authors measured the critical concentration for polymerization in the presence of ATP and ADP and found these two values to be close to each other-one requirement for treadmilling.

Very recent work from Mayer and Amman (2009) successfully purified and polymerized MreB from B. subtilis (Mayer and Amann 2009). This protein is 56\% identical and $76 \%$ similar to MreB1 from T. maritima. The kinetic behavior of this enzyme was drastically different from that of T. maritima MreB1. Polymerization of B. subtilis MreB required millimolar concentrations of divalent cations, was favored by low $\mathrm{pH}$, and was inhibited by monovalent salts and low temperatures. The authors found that B. subtilis MreB binds and hydrolyzes ATP and GTP, but surprisingly, does not require nucleotide to polymerize. Indeed, the critical concentration for polymerization was $\sim 900$ $\mathrm{nM}$ regardless of the presence or absence of nucleotide.

Bean and Amann also produced the first fluorescently labeled MreB in vitro by binding a dye to an engineered cysteine residue at position 332 (Bean and Amann 2008). Using a fluorescence-resonance energy transfer measurement between adjacent monomers in a filament, the authors found similar bulk polymerization kinetics to that measured with light scattering. Unlike the measurements from ParM, no one has yet measured the kinetics of MreB polymer elongation using fluorescence microscopy. These measurements are likely to be very important because of the combination of elongation and filament bundling seen in MreB.

Because of MreB's propensity to self-assemble laterally into bundles, many questions remain about translating in vitro kinetic data to an in vivo context. In vitro bundles can be very thick and quite crystalline. Is MreB bundled inside a cell, and if so, how thick are these bundles? Fast growing E. coli cells contain $\sim 40,000$ MreB monomers which, if fully polymerized into a single helical bundle that spans the cell, suggests a bundle thickness of greater than 50 protofilaments (Kruse et al. 2003). Assuming that these filaments are $\sim 5 \mathrm{~nm}$ in width, a tightly packed bundle would have a width of at least $50 \mathrm{~nm}$. The mechanisms by which cells control MreB filament size, geometry, and conformation remains to be discovered. Future experiments that take advantage of recent improvements in electron-microscopy or super-resolution fluorescence imaging will likely be required to address these issues.

\section{CONCLUSIONS AND OUTLOOK}

The ParM-based system of R1 plasmid segregation suggests that at least some bacterial cytoskeletons have relatively simple and specific functions. Here, regulated polymerization of ParM mechanically drives two plasmids to opposite cell poles. In contrast, the shear number of functions that have been associated with MreB suggests that the MreB situation may be far more complicated than that observed with other cytoskeletal proteins. The primary outstanding challenge for the field is to understand the mechanistic basis for these many functions and distinguish which effects are directly or indirectly mediated by MreB. It is possible that MreB carries out a small set of relatively simple 
functions that are used over and over for different purposes. For example, MreB's entire function could be to define a helical path along the long axis of rodlike cells, thereby directly or indirectly organizing the rest of the cellular components. Multiple helical structures have been identified in bacteria in the past decade. It will be important to determine whether these helices are independent such that the helix is perhaps simply an energetically favorable conformation for many structures, or whether all of these helices are ultimately patterned interdependently, either by MreB or another structure. Another outstanding question concerns the functional significance of the MreB helix. The fact that some cells transition from having helical to medial MreB suggests that different organizations of MreB filaments could play different functions, but how and why this may occur remains mysterious.

By analogy to eukaryotic actin, it has been largely assumed that MreB assembly is related to its function. However, it is possible that the eukaryotic analogy is stifling our perspective on MreB, which could be playing yet unconsidered functions. The properties of insertional polymerization, treadmilling, and structural mechanics could be combined and controlled by different proteins for different cellular purposes. Alternatively, assembly dynamics may only be important for forming the helical structure, and a multitude of MreB-binding proteins may have been adapted to tailor MreB's function for each individual activity. Indeed, eukaryotes use many regulators to control each step of actin assembly and use divergent myosins to traffic cargoes along actin. No such assembly regulators or motor proteins have thus far been identified for MreB, and it will be of interest to see if they exist and whether they are phylogenetically conserved. Advancing our understanding of MreB will thus require bringing to MreB the level of mechanistic detail that we currently enjoy for ParM. The analysis of ParM was largely driven by a bottom-up approach of reconstructing the system in vitro from its individual parts. It remains unclear whether this approach will work for more complex systems with many components and regulators. In light of the fact that a decade ago bacteria were not even thought to possess actin proteins, we now know a great deal about the bacterial actin superfamily. The prospect of combining new molecular, genetic, biophysical, and imaging approaches promises to reward the bacterial actin field with a far deeper understanding in the decade to come.

\section{REFERENCES}

Aaron M, Charbon G, Lam H, Schwarz H, Vollmer W, Jacobs-Wagner C. 2007. The tubulin homologue FtsZ contributes to cell elongation by guiding cell wall precursor synthesis in Caulobacter crescentus. Mol Microbiol 64: 938-952.

Alyahya SA, Alexander R, Costa T, Henriques AO, Emonet T, Jacobs-Wagner C. 2009. RodZ, a component of the bacterial core morphogenic apparatus. Proc Natl Acad Sci US A 106: $1239-1244$.

Bean GJ, Amann KJ. 2008. Polymerization properties of the Thermotoga maritima actin MreB: roles of temperature, nucleotides, and ions. Biochemistry 47: 826-835.

Bean GJ, Flickinger ST, Westler WM, McCully ME, Sept D, Weibel DB, Amann KJ. 2009. A22 disrupts the bacterial actin cytoskeleton by directly binding and inducing a low-affinity state in MreB. Biochemistry 48: 48524857.

Becker E, Herrera NC, Gunderson FQ, Derman AI, Dance AL, Sims J, Larsen RA, Pogliano J. 2006. DNA segregation by the bacterial actin AlfA during Bacillus subtilis growth and development. Embo J 25: 5919-5931.

Bendezu FO, de Boer PA. 2008. Conditional lethality, division defects, membrane involution, and endocytosis in mre and mrd shape mutants of Escherichia coli. J Bacteriol 190: $1792-1811$.

Bendezu FO, Hale CA, Bernhardt TG, de Boer PA. 2009. RodZ (YfgA) is required for proper assembly of the MreB actin cytoskeleton and cell shape in E. coli. EMBO J 28: 193-204.

Bork P, Sander C, Valencia A. 1992. An ATPase domain common to prokaryotic cell cycle proteins, sugar kinases, actin, and hsp70 heat shock proteins. Proc Natl Acad Sci U S A 89: 7290-7294.

Bowman GR, Comolli LR, Zhu J, Eckart M, Koenig M, Downing KH, Moerner WE, Earnest T, Shapiro L. 2008. A polymeric protein anchors the chromosomal origin/ParB complex at a bacterial cell pole. Cell 134: 945-955.

Carballido-Lopez R. 2006. The bacterial actin-like cytoskeleton. Microbiol Mol Biol Rev 70: 888-909.

Carballido-Lopez R, Errington J. 2003. The bacterial cytoskeleton: in vivo dynamics of the actin-like protein $\mathrm{Mbl}$ of Bacillus subtilis. Dev Cell 4: 19-28.

Carballido-Lopez R, Formstone A, Li Y, Ehrlich SD, Noirot P, Errington J. 2006. Actin homolog MreBH governs cell morphogenesis by localization of the cell wall hydrolase LytE. Dev Cell 11: 399-409. 
Chiu SW, Chen SY, Wong HC. 2008. Localization and expression of MreB in Vibrio parahaemolyticus under different stresses. Appl Environ Microbiol 74: 7016-7022.

Choi CL, Claridge SA, Garner EC, Alivisatos AP, Mullins RD. 2008. Protein-nanocrystal conjugates support a single filament polymerization model in R1 plasmid segregation. J Biol Chem 283: 28081-28086.

Cowles KN, Gitai Z. 2010. Surface association and the MreB cytoskeleton regulate pilus production, localization and function in Pseudomonas aeruginosa. Mol Microbiol.

Dam M, Gerdes K. 1994. Partitioning of plasmid R1. Ten direct repeats flanking the parA promoter constitute a centromere-like partition site parC, that expresses incompatibility. J Mol Biol 236: 1289-1298.

Daniel RA, Errington J. 2003. Control of cell morphogenesis in bacteria: Two distinct ways to make a rod-shaped cell. Cell 113: 767-776.

Defeu Soufo HJ, Graumann PL. 2004. Dynamic movement of actin-like proteins within bacterial cells. EMBO Rep $\mathbf{5}$ 789-794.

Defeu Soufo HJ, Graumann PL. 2005. Bacillus subtilis actinlike protein MreB influences the positioning of the replication machinery and requires membrane proteins $\mathrm{MreC} / \mathrm{D}$ and other actin-like proteins for proper localization. BMC Cell Biol 6: 10.

Defeu Soufo HJ, Reimold C, Linne U, Knust T, Gescher J, Graumann PL. 2010. Bacterial translation elongation factor EF-Tu interacts and colocalizes with actin-like MreB protein. Proc Natl Acad Sci U S A 107: 3163-3168.

Derman AI, Becker EC, Truong BD, Fujioka A, Tucey TM, Erb ML, Patterson PC, Pogliano J. 2009. Phylogenetic analysis identifies many uncharacterized actin-like proteins (Alps) in bacteria: Regulated polymerization, dynamic instability and treadmilling in Alp7A. Mol Microbiol 73: 534-552.

Divakaruni AV, Baida C, White CL, Gober JW. 2007. The cell shape proteins $\mathrm{MreB}$ and $\mathrm{MreC}$ control cell morphogenesis by positioning cell wall synthetic complexes. Mol Microbiol 66: 174-188.

Divakaruni AV, Loo RR, Xie Y, Loo JA, Gober JW. 2005. The cell-shape protein $\mathrm{MreC}$ interacts with extracytoplasmic proteins including cell wall assembly complexes in Caulobacter crescentus. Proc Natl Acad Sci 102: 18602-18607.

Doi M, Wachi M, Ishino F, Tomioka S, Ito M, Sakagami Y, Suzuki A, Matsuhashi M. 1988. Determinations of the DNA sequence of the $m r e B$ gene and of the gene products of the mre region that function in formation of the rod shape of Escherichia coli cells. J Bacteriol 170: 4619-4624.

Dye NA, Pincus Z, Theriot JA, Shapiro L, Gitai Z. 2005. Two independent spiral structures control cell shape in Caulobacter. Proc Natl Acad Sci 102: 18608-18613.

Esue O, Wirtz D, Tseng Y. 2006. GTPase activity, structure, and mechanical properties of filaments assembled from bacterial cytoskeleton protein MreB. J Bacteriol 188: 968-976.

Esue O, Cordero M, Wirtz D, Tseng Y. 2005. The assembly of MreB, a prokaryotic homolog of actin. J Biol Chem 280: $2628-2635$
Fenton AK, Lambert C, Wagstaff PC, Sockett RE. 2010. Manipulating each MreB of Bdellovibrio bacteriovorus gives diverse morphological and predatory phenotypes. J Bacteriol 192: 1299-1311.

Figge RM, Divakaruni AV, Gober JW. 2004. MreB, the cell shape-determining bacterial actin homologue, coordinates cell wall morphogenesis in Caulobacter crescentus. Mol Microbiol 51: 1321-1332.

Formstone A, Errington J. 2005. A magnesium-dependent mreB null mutant: implications for the role of $m r e B$ in Bacillus subtilis. Mol Microbiol 55: 1646-1657.

Formstone A, Carballido-Lopez R, Noirot P, Errington J, Scheffers DJ. 2008. Localization and interactions of teichoic acid synthetic enzymes in Bacillus subtilis. J Bacteriol 190: $1812-1821$.

Gan L, Chen S, Jensen GJ. 2008. Molecular organization of Gram-negative peptidoglycan. Proc Natl Acad Sci 105: 18953-18957.

Garner EC. 2008. GE Prize essay. Understanding a minimal DNA-segregating machine. Science 322: 1486-1487.

Garner EC, Campbell CS, Mullins RD. 2004. Dynamic instability in a DNA-segregating prokaryotic actin homolog. Science 306: 1021-1025.

Garner EC, Campbell CS, Weibel DB, Mullins RD. 2007. Reconstitution of DNA segregation driven by assembly of a prokaryotic actin homolog. Science 315: 1270-1274.

Gerdes K, Larsen JE, Molin S. 1985. Stable inheritance of plasmid R1 requires two different loci. J Bacteriol 161: 292-298.

Gitai Z, Dye N, Shapiro L. 2004. An actin-like gene can determine cell polarity in bacteria. Proc Natl Acad Sci 101: $8643-8648$.

Gitai Z, Dye NA, Reisenauer A, Wachi M, Shapiro L. 2005. MreB actin-mediated segregation of a specific region of a bacterial chromosome. Cell 120: 329-341.

Holmes KC, Popp D, Gebhard W, Kabsch W. 1990. Atomic model of the actin filament. Nature 347: 44-49.

Holtje JV, Heidrich C. 2001. Enzymology of elongation and constriction of the murein sacculus of Escherichia coli. Biochimie 83: 103-108.

Hu B, Yang G, Zhao W, Zhang Y, Zhao J. 2007. MreB is important for cell shape but not for chromosome segregation of the filamentous cyanobacterium Anabaena sp. PCC 7120. Mol Microbiol 63: 1640-1652.

Huang KC, Mukhopadhyay R, Wen B, Gitai Z, Wingreen NS. 2008. Cell shape and cell-wall organization in Gramnegative bacteria. Proc Natl Acad Sci 105: 19282-19287.

Iwai N, Nagai K, Wachi M. 2002. Novel S-benzylisothiourea compound that induces spherical cells in Escherichia coli probably by acting on a rod-shape-determining protein(s) other than penicillin-binding protein 2. Biosci Biotechnol Biochem 66: 2658-2662.

Jensen RB, Gerdes K. 1997. Partitioning of plasmid R1. The ParM protein exhibits ATPase activity and interacts with the centromere-like ParR-parC complex. J Mol Biol 269: 505-513.

Jensen RB, Gerdes K. 1999. Mechanism of DNA segregation in prokaryotes: ParM partitioning protein of plasmid R1 co-localizes with its replicon during the cell cycle. $E M B O$ J 18: 4076-4084. 
Jones LJ, Carballido-Lopez R, Errington J. 2001. Control of cell shape in bacteria: helical, actin-like filaments in Bacillus subtilis. Cell 104: 913-922.

Karczmarek A, Martinez-Arteaga R, Alexeeva S, Hansen FG, Vicente M, Nanninga N, den Blaauwen T. 2007. DNA and origin region segregation are not affected by the transition from rod to sphere after inhibition of Escherichia coli MreB by A22. Mol Microbiol 65: 51-63.

Kawai Y, Asai K, Errington J. 2009. Partial functional redundancy of MreB isoforms, MreB, $\mathrm{Mbl}$ and $\mathrm{MreBH}$, in cell morphogenesis of Bacillus subtilis. Mol Microbiol 73: 719-731.

Kim SY, Gitai Z, Kinkhabwala A, Shapiro L, Moerner WE. 2006. Single molecules of the bacterial actin MreB undergo directed treadmilling motion in Caulobacter crescentus. Proc Natl Acad Sci U S A 103: 10929-10934.

Komeili A, Li Z, Newman DK, Jensen GJ. 2006. Magnetosomes are cell membrane invaginations organized by the actin-like protein MamK. Science 311: 242-245.

Kruse T, Bork-Jensen J, Gerdes K. 2005. The morphogenetic MreBCD proteins of Escherichia coli form an essential membrane-bound complex. Mol Microbiol 55: 78-89.

Kruse T, Blagoev B, Lobner-Olesen A, Wachi M, Sasaki K, Iwai N, Mann M, Gerdes K. 2006. Actin homolog MreB and RNA polymerase interact and are both required for chromosome segregation in Escherichia coli. Genes Dev 20: $113-124$.

Kruse T, Moller-Jensen J, Lobner-Olesen A, Gerdes K. 2003. Dysfunctional MreB inhibits chromosome segregation in Escherichia coli. EMBO J 22: 5283-5292.

Kurner J, Frangakis AS, Baumeister W. 2005. Cryo-electron tomography reveals the cytoskeletal structure of Spiroplasma melliferum. Science 307: 436-438.

Madabhushi R, Marians KJ. 2009. Actin homolog MreB affects chromosome segregation by regulating topoisomerase IV in Escherichia coli. Mol Cell 33: 171-180.

Mauriello EM, Mouhamar F, Nan B, Ducret A, Dai D, Zusman DR, Mignot T. 2010. Bacterial motility complexes require the actin-like protein, $\mathrm{MreB}$ and the Ras homologue, MglA. EMBO J 29: 315-326.

Mayer JA, Amann KJ. 2009. Assembly properties of the Bacillus subtilis actin, MreB. Cell Motil Cytoskeleton 66: 109-118.

Mazza P, Noens EE, Schirner K, Grantcharova N, Mommaas AM, Koerten HK, Muth G, Flardh K, van Wezel GP, Wohlleben W. 2006. MreB of Streptomyces coelicolor is not essential for vegetative growth but is required for the integrity of aerial hyphae and spores. Mol Microbiol 60: $838-852$.

Michie KA, Löwe J. 2006. Dynamic filaments of the bacterial cytoskeleton. Annu Rev Biochem 75: 467-492.

Moller-Jensen J, Borch J, Dam M, Jensen RB, Roepstorff P, Gerdes K. 2003. Bacterial mitosis: ParM of plasmid R1 moves plasmid DNA by an actin-like insertional polymerization mechanism. Mol Cell 12: 1477-1487.

Moller-Jensen J, Jensen RB, Lowe J, Gerdes K. 2002. Prokaryotic DNA segregation by an actin-like filament. EMBO J 21: 3119-3127.

Munoz-Espin D, Daniel R, Kawai Y, Carballido-Lopez R, Castilla-Llorente V, Errington J, Meijer WJ, Salas M. 2009. The actin-like MreB cytoskeleton organizes viral
DNA replication in bacteria. Proc Natl Acad Sci 106: 13347-13352.

Oda T, Iwasa M, Aihara T, Maeda Y, Narita A. 2009. The nature of the globular- to fibrous-actin transition. Nature 457: 441-445.

Polka JK, Kollman JM, Agard DA, Mullins RD. 2009. The structure and assembly dynamics of plasmid actin AlfA imply a novel mechanism of DNA segregation. J Bacteriol 191: 6219-6230.

Popp D, Narita A, Oda T, Fujisawa T, Matsuo H, Nitanai Y, Iwasa M, Maeda K, Onishi H, Maeda Y. 2008. Molecular structure of the ParM polymer and the mechanism leading to its nucleotide-driven dynamic instability. $E M B O J$ 27: $570-579$.

Robertson GT, Doyle TB, Du Q, Duncan L, Mdluli KE, Lynch AS. 2007. A novel indole compound that inhibits Pseudomonas aeruginosa growth by targeting MreB is a substrate for MexAB-OprM. J Bacteriol 189: 6870-6881.

Rokney A, Shagan M, Kessel M, Smith Y, Rosenshine I, Oppenheim AB. 2009. E. coli transports aggregated proteins to the poles by a specific and energy-dependent process. J Mol Biol 392: 589-601.

Salje J, Zuber B, Lowe J. 2009. Electron cryomicroscopy of E. coli reveals filament bundles involved in plasmid DNA segregation. Science 323: 509-512.

Savage DF, Afonso B, Chen AH, Silver PA. 2010. Spatially ordered dynamics of the bacterial carbon fixation machinery. Science 327: 1258-1261.

Scheffers DJ, Jones LJ, Errington J. 2004. Several distinct localization patterns for penicillin-binding proteins in Bacillus subtilis. Mol Microbiol 51: 749-764.

Shebelut CW, Jensen RB, Gitai Z. 2009. Growth conditions regulate the requirements for Caulobacter chromosome segregation. J Bacteriol 191: 1097-1100.

Shih YL, Kawagishi I, Rothfield L. 2005. The MreB and Min cytoskeletal-like systems play independent roles in prokaryotic polar differentiation. Mol Microbiol 58: 917-928.

Shiomi D, Sakai M, Niki H. 2008. Determination of bacterial rod shape by a novel cytoskeletal membrane protein. EMBO J 27: 3081-3091.

Slovak PM, Wadhams GH, Armitage JP. 2005. Localization of MreB in Rhodobacter sphaeroides under conditions causing changes in cell shape and membrane structure. J Bacteriol 187: 54-64.

Soufo HJ, Graumann PL. 2003. Actin-like proteins MreB and $\mathrm{Mbl}$ from Bacillus subtilis are required for bipolar positioning of replication origins. Curr Biol 13: 1916-1920.

Srinivasan R, Mishra M, Murata-Hori M, Balasubramanian MK. 2007. Filament formation of the Escherichia coli actin-related protein, MreB, in fission yeast. Curr Biol 17: $266-272$.

Srivastava P, Demarre G, Karpova TS, McNally J, Chattoraj DK. 2007. Changes in nucleoid morphology and origin localization upon inhibition or alteration of the actinhomolog, MreB, of Vibrio cholerae. J Bacteriol.

Takacs CN, Poggio S, Charbon G, Pucheault M, Vollmer W, Jacobs-Wagner C. 2010. MreB drives de novo rod morphogenesis in Caulobacter crescentus via remodeling of the cell wall. J Bacteriol 192: 1671-1684. 
J.W. Shaevitz and Z. Gitai

Tiyanont K, Doan T, Lazarus MB, Fang X, Rudner DZ Walker S. 2006. Imaging peptidoglycan biosynthesis in Bacillus subtilis with fluorescent antibiotics. Proc Natl Acad Sci U S A 103: 11033-11038.

Touhami A, Jericho MH, Beveridge TJ. 2004. Atomic force microscopy of cell growth and division in Staphylococcus aureus. J Bacteriol 186: 3286-3295.

van den Ent F, Amos LA, Lowe J. 2001. Prokaryotic origin of the actin cytoskeleton. Nature 413: 39-44.

van den Ent F, Johnson CM, Persons L, de Boer P, Lowe J. 2010. Bacterial actin MreB assembles in complex with cell shape protein RodZ. EMBO J 29: 1081-1090.

van den Ent F, Lowe J. 2000. Crystal structure of the cell division protein FtsA from Thermotoga maritima. EMBO 19: $5300-5307$.

van den Ent F, Leaver M, Bendezu F, Errington J, de Boer P, Lowe J. 2006. Dimeric structure of the cell shape protein MreC and its functional implications. Mol Microbiol 62: $1631-1642$.

van den Ent F, Moller-Jensen J, Amos LA, Gerdes K, Lowe J. 2002. F-actin-like filaments formed by plasmid segregation protein ParM. EMBO J 21: 6935-6943.
Vats P, Shih YL, Rothfield L. 2009. Assembly of the MreB-associated cytoskeletal ring of Escherichia coli. Mol Microbiol 72: 170-182.

Wagner JK, Galvani CD, Brun YV. 2005. Caulobacter crescentus requires RodA and MreB for stalk synthesis and prevention of ectopic pole formation. J Bacteriol 187: 544-553.

Waidner B, Specht M, Dempwolff F, Haeberer K, Schaetzle S, Speth V, Kist M, Graumann PL. 2009. A novel system of cytoskeletal elements in the human pathogen Helicobacter pylori. PLoS Pathog 5: e1000669.

Wang S, Arellano-Santoyo H, Combs PA, Shaevitz JW. 2010. An actin-like cytoskeleton contributes to cell mechanics in bacteria. Proc Natl Acad Sci 107: 9182-9185.

White CL, Kitich A, Gober JW. 2010. Positioning cell wall synthetic complexes by the bacterial morphogenetic proteins MreB and MreD. Mol Microbiol 76: 616-633.

Yao X, Jericho M, Pink D, Beveridge T. 1999. Thickness and elasticity of gram-negative murein sacculi measured by atomic force microscopy. J Bacteriol 181: 6865-6875.

Young KD. 2006. The selective value of bacterial shape. Microbiol Mol Biol Rev 70: 660-703. 


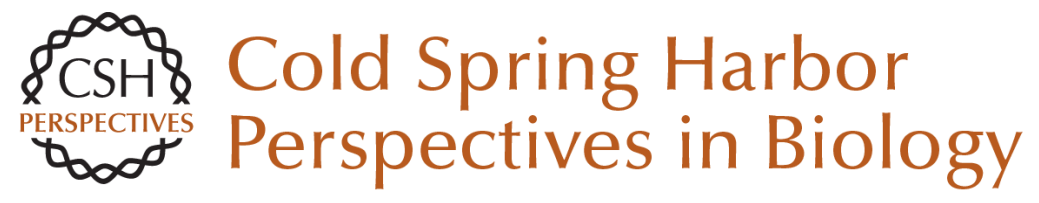

\section{The Structure and Function of Bacterial Actin Homologs}

Joshua W. Shaevitz and Zemer Gitai

Cold Spring Harb Perspect Biol 2010; doi: 10.1101/cshperspect.a000364 originally published online July 14,2010

\section{Subject Collection Cell Biology of Bacteria}

\section{Electron Cryotomography}

Elitza I. Tocheva, Zhuo Li and Grant J. Jensen

Protein Subcellular Localization in Bacteria David Z. Rudner and Richard Losick

Poles Apart: Prokaryotic Polar Organelles and Their Spatial Regulation

Clare L. Kirkpatrick and Patrick H. Viollier

Myxobacteria, Polarity, and Multicellular

Morphogenesis

Dale Kaiser, Mark Robinson and Lee Kroos

Membrane-associated DNA Transport Machines

Briana Burton and David Dubnau

The Bacterial Cell Envelope

Thomas J. Silhavy, Daniel Kahne and Suzanne Walker

Cell Biology of Prokaryotic Organelles Dorothee Murat, Meghan Byrne and Arash Komeili

Bacterial Chromosome Organization and

\section{Segregation}

Esteban Toro and Lucy Shapiro
Cyanobacterial Heterocysts
Krithika Kumar, Rodrigo A. Mella-Herrera and
James W. Golden

Synchronization of Chromosome Dynamics and

Cell Division in Bacteria Martin Thanbichler

Automated Quantitative Live Cell Fluorescence

Microscopy

Michael Fero and Kit Pogliano

The Structure and Function of Bacterial Actin

Homologs Joshua W. Shaevitz and Zemer Gitai

\section{Biofilms}

Daniel López, Hera Vlamakis and Roberto Kolter

Bacterial Nanomachines: The Flagellum and Type

III Injectisome

Marc Erhardt, Keiichi Namba and Kelly T. Hughes

Single-Molecule and Superresolution Imaging in

Live Bacteria Cells Julie S. Biteen and W.E. Moerner

For additional articles in this collection, see http://cshperspectives.cshlp.org/cgi/collection/

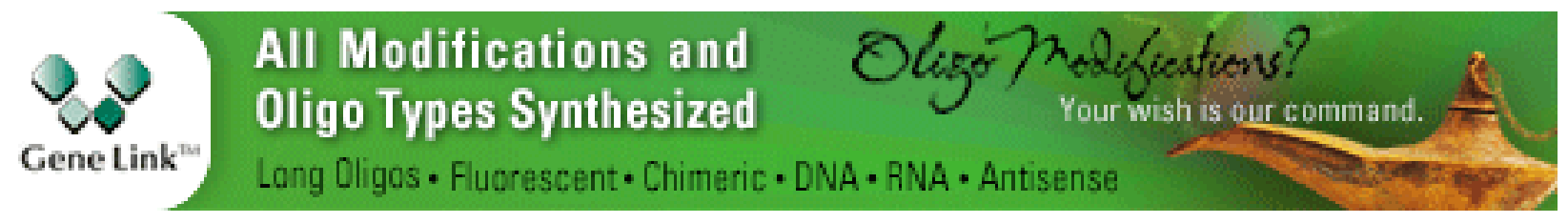

Copyright @ 2010 Cold Spring Harbor Laboratory Press; all rights reserved 